\title{
Role of bardoxolone methyl, a nuclear factor erythroid 2-related factor 2 activator, in aldosterone- and salt-induced renal injury
}

\author{
Mikako Hisamichi $^{1}$, Atsuko Kamijo-Ikemori ${ }^{1,2}$, Takeshi Sugaya ${ }^{1}$, Seiko Hoshino ${ }^{2}$, Kenjiro Kimura ${ }^{3}$ \\ and Yugo Shibagaki ${ }^{1}$
}

The aim of this study was to investigate the renoprotective effect of bardoxolone methyl (BM), a nuclear factor erythroid 2-related factor 2 (Nrf2) activator with an antioxidant effect, in a salt-sensitive hypertension model induced by aldosterone (Ald) and salt. Tubulointerstitial damage with urinary liver-type fatty acid-binding protein (L-FABP) was evaluated using human L-FABP chromosomal transgenic $\left(\mathrm{L}-F A B P^{+/-}\right)$male mice. The mice in the Ald group $(n=7)$ received systemic Ald infusions via an osmotic minipump and were given $1 \% \mathrm{NaCl}$ water for 35 days. Those in the Ald-BM group $(n=8)$ were administered BM intraperitoneally in addition to an injection of Ald and salt. The dose of BM was gradually increased every 7 days up to $10 \mathrm{mg} \mathrm{kg}^{-1}$ per day, which was maintained for 14 days. The administration of BM significantly increased renal expression of the Nrf2 target antioxidant gene. Tubulointerstitial damage was significantly ameliorated in the Ald-BM group compared to the Ald group. The increase in reactive oxygen species (ROS) and upregulation of angiotensinogen expression in the kidneys of the Ald group was significantly prevented in the Ald-BM group. The upregulation of human L-FABP expression induced in the kidneys and increase in urinary L-FABP in the Ald group were significantly suppressed by BM administration. In conclusion, BM ameliorated tubulointerstitial damage in the Ald- and salt-induced hypertension model through suppression of both ROS production and intrarenal renin-angiotensin system activation. Urinary L-FABP may be a useful marker reflecting the therapeutic efficacy of BM.

Hypertension Research (2018) 41, 8-17; doi:10.1038/hr.2017.83; published online 5 October 2017

Keywords: aldosterone (Ald); bardoxolone methyl (BM); liver-type fatty acid-binding protein (L-FABP); nuclear factor erythroid 2-related factor 2 (Nrf2); oxidative stress

\section{INTRODUCTION}

Salt-sensitive hypertension induces redundant activation of the reninangiotensin-aldosterone (Ald) system. ${ }^{1}$ Accumulating evidence shows that Ald, an end product of that system, plays a key role in the progression of chronic kidney disease (CKD) due to salt-sensitive hypertension, ${ }^{2-4}$ and that mineralocorticoid receptor (MR) blockers, which inhibit the injurious effect of Ald on the kidney, contribute to a reduction in albuminuria and proteinuria in CKD patients. ${ }^{5,6} \mathrm{MR}$ blockers are therefore considered to play a crucial role in preventing the progression of CKD with hypertension. However, treatment with MR blockers can induce hyperkalemia, which is a concern in patients with renal dysfunction and requires caution in prescribing MR blockers for advanced CKD patients.

Ald is associated with cellular oxidative stress, ${ }^{7,8}$ inducing both glomerular and tubulointerstitial injury in the salt-sensitive hypertension model. ${ }^{9-12}$ As tubulointerstitial damage is strongly associated with the prognosis of $\mathrm{CKD},{ }^{13,14}$ a new strategy with antioxidant activity is needed for preventing the progression of such damage due to hypertension.

Liver-type fatty acid-binding protein (L-FABP), a small protein with a molecular weight of $14 \mathrm{kDa}$, is expressed in proximal tubules of the human kidneys and has an antioxidant activity through the inactivation of free radicals by its methionine and cysteine amino acids. ${ }^{15}$ As L-FABP is not expressed in mouse kidneys, ${ }^{16}$ we have generated chromosomal transgenic mice in which human L-FABP was expressed in the proximal tubules by microinjecting human L-FABP genomic DNA, including its promoter region. ${ }^{17}$ Our previous study using these mice showed that upregulated expression of human L-FABP in the proximal tubules suppressed the progression of tubulointerstitial injury through reducing oxidative stress in an Ald- and salt-induced hypertension model, ${ }^{18}$ although this did not result in complete renoprotection. On the other hand, levels of urinary human L-FABP, one of the tubular markers, reflected the amelioration of tubulointerstitial damage by treatment with MR blockers in the model ${ }^{18}$

${ }^{1}$ Department of Anatomy, Kanagawa, Japan; ${ }^{2}$ Department of Anatomy, St. Marianna University School of Medicine, Kanagawa, Japan and ${ }^{3} J C H O$ Tokyo Takanawa Hospital, Tokyo, Japan

Correspondence: Dr A Kamijo-Ikemori, Department of Anatomy, 2-16-1 Sugao, Miyamae-Ku, Kawasaki 216-8511, Japan.

E-mail: a2kamijo@marianna-u.ac.jp

Received 30 March 2017; revised 6 July 2017; accepted 31 July 2017; published online 5 October 2017 
and urinary L-FABP may be a useful marker for the evaluation of tubulointerstital damage in the Ald- and salt-induced hypertension model.

The activation of nuclear factor erythroid 2-related factor 2 (Nrf2) has antioxidant and anti-inflammatory effects. Protective effects of Nrf2 activators have been reported for organs such as the lung, liver, gastrointestinal tract and nervous system. ${ }^{19}$ Their renoprotective effects have been verified using Nrf2-deficient mice in, for instance, diabetic nephropathy, ${ }^{20}$ lupus-like autoimmune nephritis ${ }^{21}$ and ischemic reperfusion injury. ${ }^{22}$ Bardoxolone methyl (BM), one such Nrf2 activator, has been reported in clinical practice to have a beneficial effect on the renal function of patients with type 2 diabetic nephropathy. ${ }^{23}$ In hypertension models, Nrf2 activators were reported to be related to improvement of vascular function via antioxidant property. ${ }^{24}$ Its effects might therefore be expected to extend to tubulointerstitial injury due to salt-sensitive hypertension.

The aim of this study was to investigate the renoprotective effect of $\mathrm{BM}$ through its antioxidative mechanism in an Ald- and salt-induced hypertension model under the presence of human L-FABP expression in the proximal tubules. In addition, we evaluated how the dynamics of human L-FABP in the kidneys changed with the administration of $\mathrm{BM}$ and the utility of urinary human L-FABP as an indicator of the renoprotective effect of BM.

\section{METHODS}

\section{Animals}

The studies were conducted in accordance with the St Marianna University School of Medicine's Institutional Guide for Animal Experiments and the Guide for the Care and Use of Laboratory Animals. Human L-FABP chromosomal transgenic mice of C57/BL6 background (L-FABP ${ }^{+-}$) (World Intellectual Property Organization patent WO0073791) were generated by microinjection of the genomic DNA of human L-FABP including its promoter region as described previously. ${ }^{17}$ Therefore, it seems possible that the transcription of the human $L-F A B P$ gene in the kidneys of the L-FABP ${ }^{+/}$ mice may be regulated by the same mode in that of human. The experiment used 8 to 10 -week-old male L-FABP ${ }^{+/-}$mice $(n=25)$. Surgical procedure was performed under appropriate anesthesia and animal suffering was avoided at each stage of the experiments.

\section{Experimental design}

The L-FABP ${ }^{+/-}$mice were divided into four groups. The Ald group $(n=7)$ received a systemic Ald infusion $\left(0.125 \mu \mathrm{g} \mathrm{kg}^{-1} \mathrm{~min}^{-1}\right.$, A9477; Sigma-Aldrich, St Louis, MO, USA) using an osmotic minipump (Alzet 1004 for 28 days and 1007D for 7 days; Durect, Cupertino, CA, USA) and was given $1 \% \mathrm{NaCl}$ water for 35 days. The Ald was dissolved in polyethylene glycol (PEG 300; SigmaAldrich Japan, Tokyo, Japan) and was infused via an implanted osmotic minipump into the subcutaneous space, while the mice were anesthetized with isoflurane. As the phase II clinical trial to determine the effect of BM (RTA402) on glomerular filtration rate in patients with type 2 diabetes and CKD (TUBAKI study) has been performed in Japan, the Ald-BM group $(n=8)$ received BM (RTA 402) (AdooQ Bioscience, Irvine, CA, USA) intraperitoneally in addition to an injection of both Ald and salt. As mice in our preliminary study died when the daily dose of BM was $10 \mathrm{mg} \mathrm{kg}^{-1}$ right from the start of the study, in the present study we increased the daily dose from 1.25 to $10 \mathrm{mg} \mathrm{kg}^{-1}$ in steps every 7 days as follows: $1.25 \mathrm{mg} \mathrm{kg}^{-1}$ for 7 days, $2.5 \mathrm{mg} \mathrm{kg}^{-1}$ for 7 days, $5 \mathrm{mg} \mathrm{kg}^{-1}$ for 7 days and $10 \mathrm{mg} \mathrm{kg}^{-1}$ for 14 days. The control group $(n=5)$ was given only a vehicle and the control-BM group $(n=5)$ was given the vehicle plus the BM in the same doses as the BM group.

On day 35, blood was drawn from the inferior vena cava of all mice and the kidneys were removed while the mice were under intraperitoneal anesthesia with pentobarbital at $75 \mathrm{mg} \mathrm{kg}^{-1}$ body weight. Part of the kidney was snapfrozen in liquid nitrogen for protein and mRNA analyses, and unfixed frozen cross-sections were stained. The remainder was fixed in methyl Carnoy's solution or $10 \%$ buffered formalin (Wako Pure Chemical Industries, Osaka, Japan).

\section{Blood pressure}

Blood pressure was measured in conscious, restrained mice by using a tail-cuff apparatus (Softron BP-98A; Softron, Tokyo, Japan). ${ }^{25}$ Systolic blood pressure (SBP) values were derived from the mean of three measurements per animal on days 0,14 and 35 .

\section{Serum and urinary biochemistry}

For urine collection on day 35, all of the mice were housed overnight individually in metallic cages with free access to $1 \% \mathrm{NaCl}$ water or water. The sediment was removed from the urine samples by centrifugation at 15,000 r.p.m. for $5 \mathrm{~min}$. Serum and urinary creatinine levels were measured by an enzymatic method (Nescoat VL II CRE; Alfresa Pharma, Osaka, Japan). ${ }^{25}$ Serum and urinary sodium were measured using an electrode method provided by the clinical laboratory testing services of SRL. Urinary parameters were reported as ratios relative to the urinary creatinine levels. Albuminuria was determined using the Albuwell assay (Exocell, Philadelphia, PA, USA). Urinary L-FABP was measured by a two-step sandwich enzyme-linked immunosorbent assay (L-FABP ELISA kit; CMIC, Tokyo, Japan). ${ }^{17}$

\section{Renal histological and morphometric analyses}

For light microscopic analysis, the kidneys were dehydrated and embedded in paraffin. Serial sections $2 \mu \mathrm{m}$-thick were obtained for conventional histological assessment, such as periodic acid-Schiff staining and Masson's trichrome staining, and for immunohistochemistry. In the tissue sections stained with periodic acid-Schiff staining, tubular injury was categorized as tubular dilation with epithelial atrophy, brush border loss and the presence of cast. Tissue fibrosis was defined as accumulation of extracellular matrix shown in blue in Masson's trichrome staining assessed under $\times 100$ magnification. Ten non-overlapping fields from the cortical and outer medullary areas were selected under $\times 100$ magnification and the regions with tubular injury or fibrosis, as well as the entire cortical and outer medullary areas, were measured with WinROOF image analysis software version 6.1 (Mitani Co., Tokyo, Japan). The degrees of tubular injury and fibrosis in each region were expressed as the ratio of the area of injury or fibrosis relative to the entire area. These histological evaluations were performed in a blinded manner by one observer.

\section{Immunohistological analysis}

Tissues fixed in methyl Carnoy's solution were embedded in paraffin. An indirect immunoperoxidase method was used to identify the antigens, as described previously. ${ }^{26,27}$ Type I and type III collagens were identified using rabbit polyclonal antibodies (Cedarlane Laboratories Ltd, Burlington, ON, Canada). Macrophages were identified using a rat monoclonal antibody against F4/80 (BMA Biomedicals, Augst, Switzerland). The primary antibodies were diluted with DAKO Antibody Diluent with Background Reducing Components (S3022, Dako Japan, Tokyo, Japan). Ten non-overlapping fields from the cortical and outer medullary areas were selected under $\times 100$ magnification and areas positively stained for type I and type III collagens were measured with WinROOF and expressed as ratios relative to the total cortical and outer medullary areas. Similarly, the degree of macrophage infiltration in the cortical and outer medullary areas was expressed as a ratio of the area positively stained for F4/80 relative to the entire area.

Tissues fixed in formalin solution were also embedded in paraffin and Nrf2 was identified using a rabbit monoclonal antibody against Nrf2 (sc-13032X; Santa Cruz Biotechnology, Santa Cruz, CA, USA), according to the method previously described. ${ }^{28}$ The primary antibody was diluted with DAKO Antibody Diluent with Background Reducing Components (S3022, Dako Japan). For the staining with anti-Nrf2 antibody, antigen retrieval was performed using a microwave oven to heat the slide for $15 \mathrm{~min}$ in $\mathrm{PH} 6.0$ citric acid buffer. The degree of $\mathrm{Nrf2}$ expression in the cortical and outer medullary areas was expressed as the ratio of the area positively stained for Nrf2 relative to the entire area, as for the other staining described earlier. 


\section{In situ detection of ROS production}

To assess the kidneys' production of reactive oxygen species (ROS) in situ, we stained unfixed frozen cross sections from the kidneys with $5 \mu \mathrm{M}$ dihydroethidium (DHE, Thermo Fisher Scientific, Inc., Waltham, MA, USA) for $30 \mathrm{~min}$ in a dark, humidified chamber at $37^{\circ} \mathrm{C}$. ROS generation was labeled with red fluorescence and visualized by fluorescence microscopy. ${ }^{18,29,30}$ The DHE fluorescence intensities (exposure time: $200 \mathrm{~ms}$ ) of kidney cross-sections were examined in ten nonoverlapping fields from the cortical areas and were expressed as the ratio of the positively stained area of DHE relative to the entire cortical area under $\times 200$ magnification measured with WinROOF.

\section{Real-time quantitative PCR analysis}

Total RNA was extracted from frozen kidneys and reverse transcribed as described previously. ${ }^{26,27}$ Real-time quantitative PCR analysis was performed using a TaqMan StepOnePlus system (Thermo Fisher Scientific) to measure the mRNA levels of Nrf2, $\mathrm{NAD}(\mathrm{P}) \mathrm{H}$ quinone oxidoreductase-1, glutathione $S$ transferase $\mu 1$, glutathione $S$-transferase $\alpha 4$, monocyte chemoattractant protein1 (MCP-1), regulated on activation, normal $\mathrm{T}$ cell expressed and secreted (RANTES), $\alpha-1$ collagen type I and type III, human L-FABP and glyceraldehyde-3-phosphate dehydrogenase. The expression levels of these transcripts in each sample were normalized to the glyceraldehyde-3phosphate dehydrogenase expression levels.

\section{Measurement of MCP-1 and human L-FABP by enzyme-linked immunosorbent assay}

Proteins were extracted from frozen kidneys and protein concentration was measured as described previously. ${ }^{25}$ The inflammatory cytokine MCP-1 and human L-FABP were measured using enzyme-linked immunosorbent assay

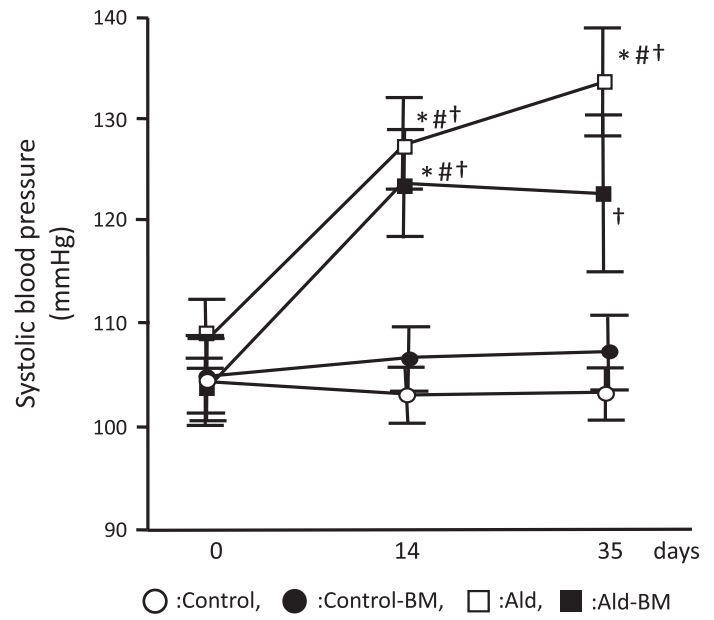

Figure 1 Time-related changes in SBP on days 0,14 and $35 .{ }^{*} P<0.05$ vs the control group on the same day; ${ }^{\#} P<0.05$ vs the control-BM group on the same day; ${ }^{\dagger} P<0.05$ vs the same group on day 0 . Abbreviations: Ald, aldosterone; BM, bardoxolone methyl.
(R\&D Systems, Minneapolis, MN, USA for MCP-1; CMIC for human L-FABP). ${ }^{25}$ The concentrations of MCP-1 and human L-FABP were corrected for total protein concentration.

\section{Statistical analysis}

Values are expressed as means \pm s.e. Statistical significance was set at $P<0.05$. The Wilcoxon signed-rank test for paired data was used for comparisons. The four groups were analyzed by Kruskal-Wallis one-way analysis of variance followed by Mann-Whitney $U$-tests. To assess the correlation of two parameters, Spearman's rank correlation coefficient was used to evaluate nonparametric data. Analyses were performed using JMP 11.2.1 (SAS Institute, Cary, NC, USA).

\section{RESULTS}

\section{Systolic blood pressure}

SBP was similar among the four groups on day 0; by days 14 and 35, it had increased significantly in the Ald and Ald-BM groups (both $P<0.05)$. In the Ald group, SBPs were significantly higher than in the control and control-BM groups on days 14 and $35 \quad(P<0.05)$ (Figure 1). In the Ald-BM group, SBPs were significantly higher than in the control and control-BM groups on day $14(P<0.05)$ (Figure 1$)$. SBPs of the Ald-BM group were similar to those of the Ald group on days 14 and 35 (Figure 1).

\section{Body weight and renal weight}

Body weight was similar between the four groups on day 0 . By day 35 , body weights had significantly increased in the control and Ald groups (Table 1). Renal weights on day 35 were significantly higher in the Ald and Ald-BM groups than in the control and control-BM groups (Table 1).

\section{Serum and urinary biochemistry}

The levels of serum creatinine were similar between the four groups on day 35 (Table 1). Serum sodium level was significantly higher in the Ald-BM group than in the other groups $(P<0.05)$ (Table 1$)$. Although urinary sodium levels were significantly higher in the Ald and Ald-BM groups than in the control and control-BM groups $(P<0.05)$ (Table 1$)$, these levels were significantly higher in the Ald-BM group than in the Ald group $(P<0.05)$.

By day 35 , the levels of urinary albumin were significantly higher in the Ald and Ald-BM groups than in the control and control-BM groups (Figure 2). The levels in the Ald-BM group were significantly lower than those in the Ald group $(P<0.05)$.

Table 1 Time-related changes in body weight, findings of renal weight, serum sodium concentration and urinary excretion of sodium on day 35.

\begin{tabular}{|c|c|c|c|c|c|c|}
\hline & \multicolumn{2}{|c|}{ Body weight } & \multirow[b]{2}{*}{ Renal weight, $g$} & \multicolumn{2}{|c|}{ Serum } & \multirow[b]{2}{*}{ Urinary sodium, $m E q$ per $m g \mathrm{Cr}$} \\
\hline & Day $O \mathrm{~g}$ & Day $35 \mathrm{~g}$ & & Creatinine, $\mathrm{mg} \mathrm{dl^{-1 }}$ & Sodium, $m E q I^{-1}$ & \\
\hline Control & $25.7 \pm 0.4$ & $27.5 \pm 0.3^{\dagger}$ & $0.17 \pm 0.01$ & $0.30 \pm 0.13$ & $149.4 \pm 1.6$ & $0.33 \pm 0.03$ \\
\hline Control-BM & $25.8 \pm 0.6$ & $26.6 \pm 0.5$ & $0.18 \pm 0.01$ & $0.19 \pm 0.04$ & $151.6 \pm 1.4$ & $0.38 \pm 0.05$ \\
\hline Ald & $25.8 \pm 0.3$ & $29.0 \pm 0.7^{\dagger}$ & $0.24 \pm 0.01^{*, \#}$ & $0.22 \pm 0.03$ & $154.4 \pm 1.7$ & $2.84 \pm 0.45^{*, \#}$ \\
\hline Ald-BM & $24.8 \pm 0.4$ & $26.3 \pm 1.2$ & $0.23 \pm 0.01^{* \#}$ & $0.20 \pm 0.07$ & $161.9 \pm 2.1^{*, \#, a}$ & $1.37 \pm 0.29 *, \#, a$ \\
\hline
\end{tabular}

Abbreviations: Ald, aldosterone; BM, bardoxolone methyl; Cr, creatinine.

Values are means \pm s.e.

${ }^{*} P<0.05$ vs the control group on the same day; ${ }^{\#} P<0.05$ vs the control-BM group on the same day; ${ }^{\dagger} P<0.05$ vs the same group on day $0 ;{ }^{\circledR} P<0.05$ vs the Ald-BM group on the same day. 
Gene expressions of Nrf2 and Nrf2 target antioxidant genes, and the immunohistological analysis for Nrf2

The mRNA levels of Nrf2 were significantly lower in the control-BM and Ald-BM groups than in the control and Ald groups (Figure 3a). The levels in the Ald and control groups were similar.

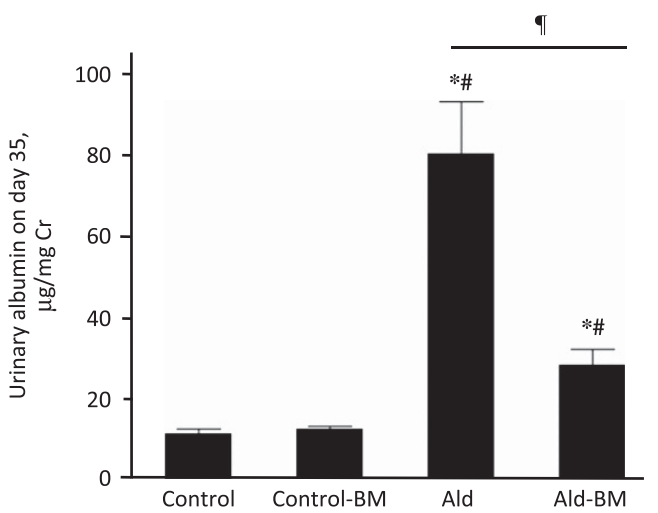

Figure 2 Urinary albumin excretion on day 35. Values are mean \pm s.e. ${ }^{*} P<0.05$ vs the control group; ${ }^{\#} P<0.05$ vs the control-BM group; ${ }^{\mathbb{T}} P<0.05$ vs the Ald group. Abbreviations: Ald, aldosterone; BM, Bardoxolone methyl.
The mRNA expressions of the Nrf2 target antioxidant genes $(\mathrm{NAD}(\mathrm{P}) \mathrm{H}$ quinone oxidoreductase-1, glutathione $S$-transferase $\mu 1$ and glutathione $S$-transferase $\alpha 4$ ) were significantly higher in the control-BM and Ald-BM groups than in the control and Ald groups (Figures $3 \mathrm{~b}-\mathrm{d}$ ). The levels in the Ald-BM and control-BM groups were similar.

The expression of Nrf2 was observed in the nucleus and cytoplasm of the tubular cells (Figure 3e). The degree of Nrf2 positive area tended to be higher in the Ald-BM group than in the Ald group $(P=0.15$, Figure $3 \mathrm{f})$ and in the control-BM group than in the control group $(P=0.06)$.

Renal expression of MCP-1 and immunohistological analysis for macrophage infiltration

The gene (Figure 4a) and protein (Figure 4b) expression levels of MCP-1 in the kidneys were significantly higher in the Ald and Ald-BM groups than in the control and control-BM groups. The renal expressions of MCP-1 were significantly lower in the Ald group than in the Ald-BM group (Figures 4a and b).

The levels of macrophage infiltration were significantly higher in the Ald group than in the control and control-BM groups, and significantly higher in the Ald-BM group than in the control-BM group (Figures $4 \mathrm{c}$ and $\mathrm{d}$ ). The levels in the Ald-BM group were significantly lower compared with those in the Ald group (Figures $4 \mathrm{c}$ and $\mathrm{d}$ ). a

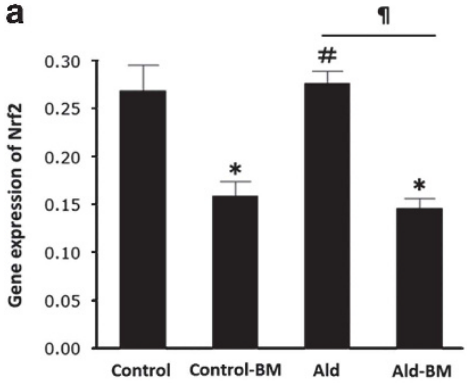

C

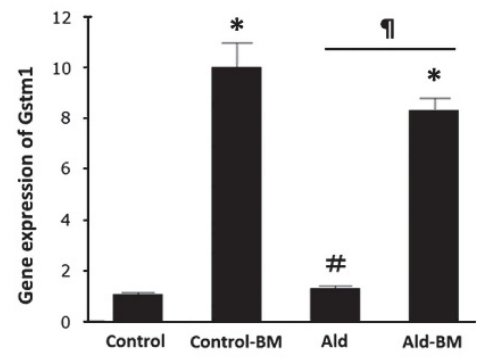

e

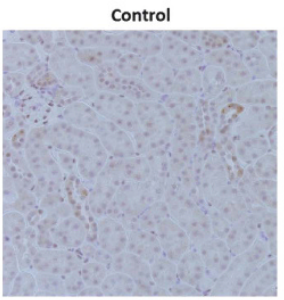

Control-BM

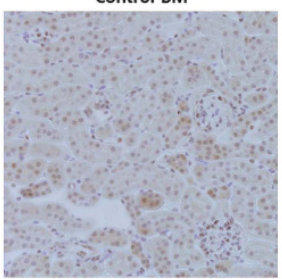

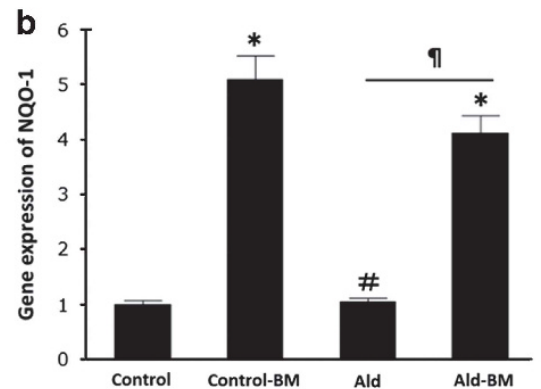

ก

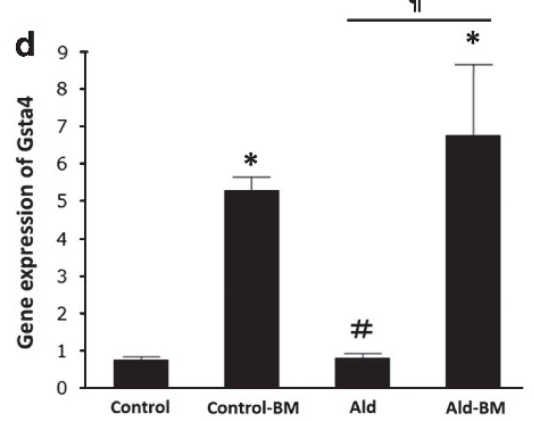

Ald

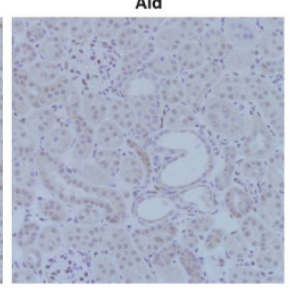

Ald-BM

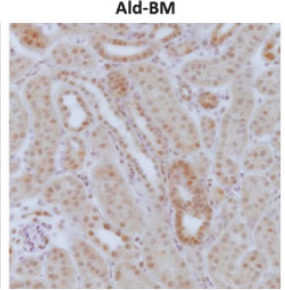

f

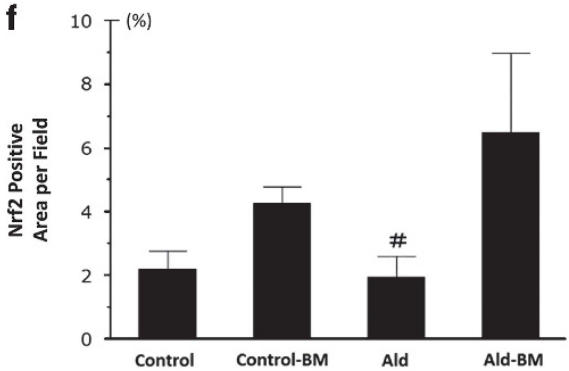

Figure 3 mRNA transcript expression of Nrf2 (a) and the Nrf2 target antioxidant genes NQO-1 (b), Gstm1 (c) and Gsta4 (d). Immunohistological staining for Nrf2 (e) and positively stained areas were assessed quantitatively (f). Original magnification, $\times 100$. ${ }^{*} P<0.05$ vs the control group; ${ }^{\#} P<0.05$ vs the control-BM group; ${ }^{\mathbb{P}} P<0.05$ vs the Ald group. Abbreviations: Ald, aldosterone; BM, bardoxolone methyl; Gsta4, glutathione $S$-transferase $\alpha 4$; Gstm1, glutathione $S$-transferase $\mu 1$; Nrf2, nuclear factor erythroid 2-related factor 2; NQO-1, NAD(P)H quinone oxidoreductase-1. 


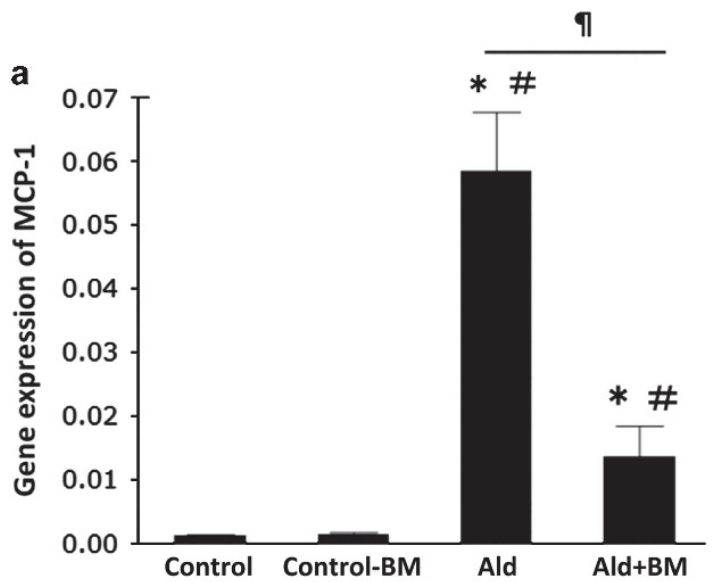

C

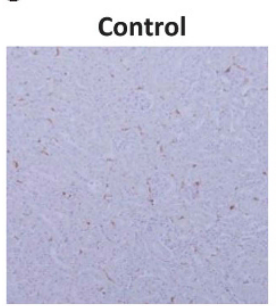

Control-BM

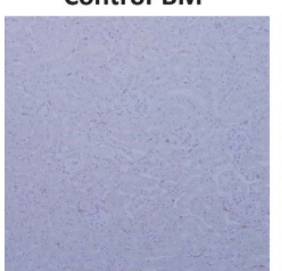

Ald

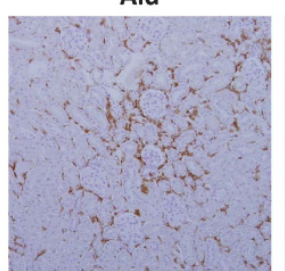

b

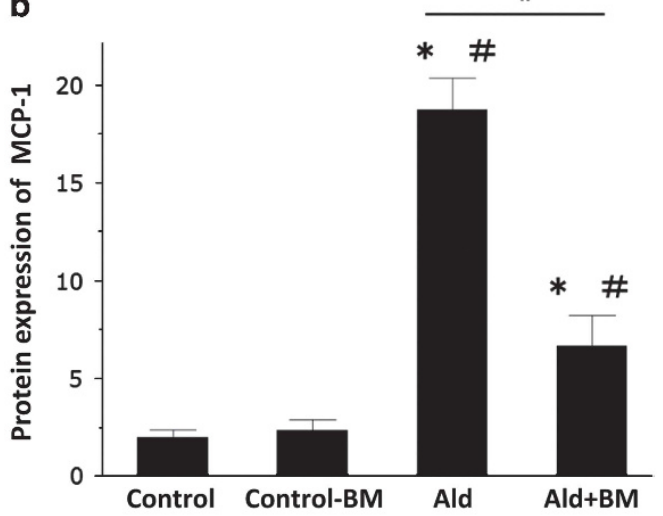

Ald+BM

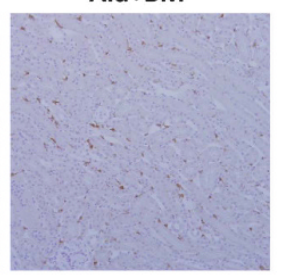

d

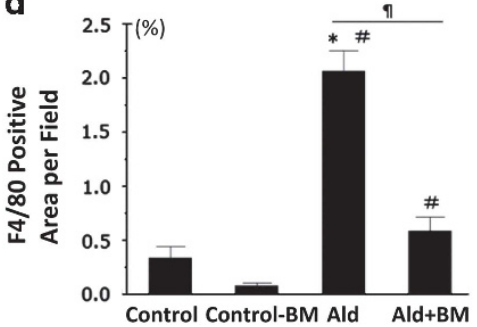

Figure 4 mRNA transcript expression of MCP-1 (a) and the protein expression of MCP-1 (b). Immunohistological staining for macrophages using an antibody against F4/80 (c) and the positively stained areas were assessed quantitatively (d). Original magnification, $\times 100$. ${ }^{*} P<0.05$ vs the control group; ${ }^{\#} P<0.05$ vs the control-BM group; ${ }^{\top} P<0.05$ vs the Ald group. Abbreviations: Ald, aldosterone; BM, bardoxolone methyl; MCP-1, monocyte chemoattractant protein-1.

a

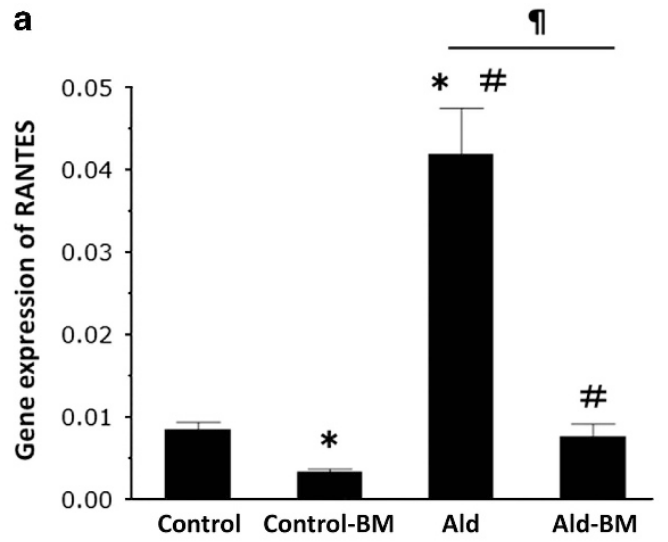

b

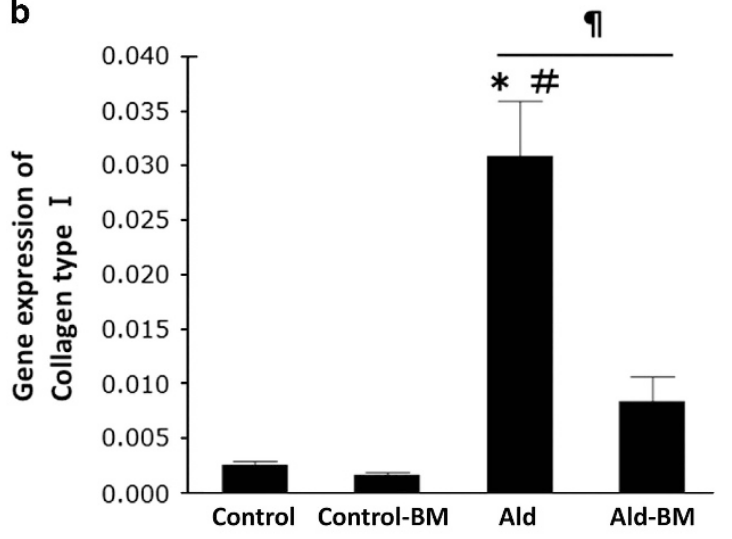

C

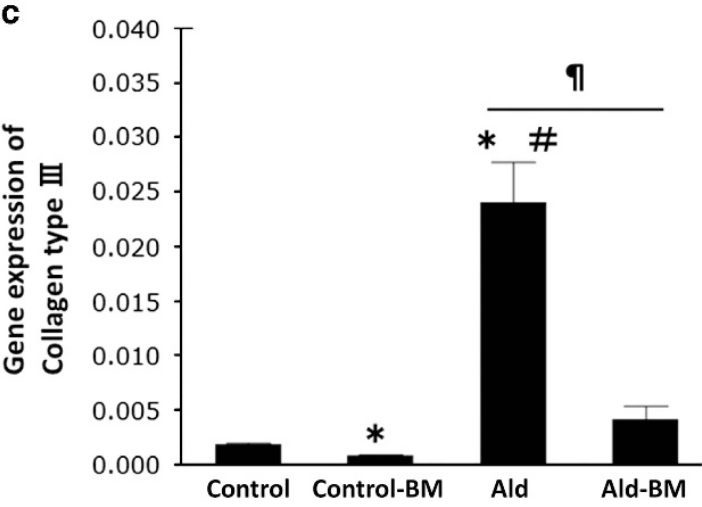

Figure 5 mRNA transcript expression of RANTES (a), $\alpha-1$ collagen type $\alpha$ (b) and $\alpha-1$ collagen type $\beta$ (c). * $P<0.05$ vs the control group; $\# P<0.05$ vs the control-BM group; ${ }^{\top} P<0.05$ vs the Ald group. Abbreviations: Ald, aldosterone; BM, bardoxolone methyl; RANTES, regulated on activation, normal T cell expressed and secreted. 
a

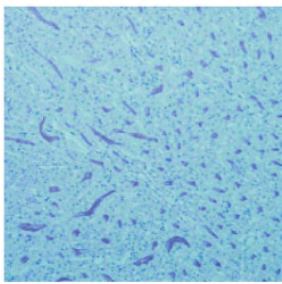

c

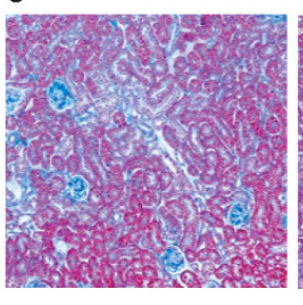

e
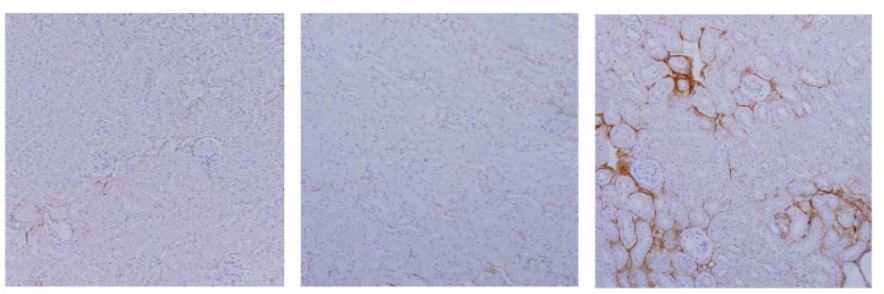

g

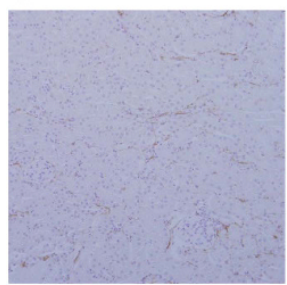

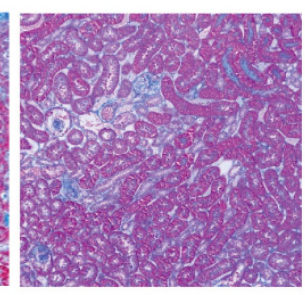

Control-BM
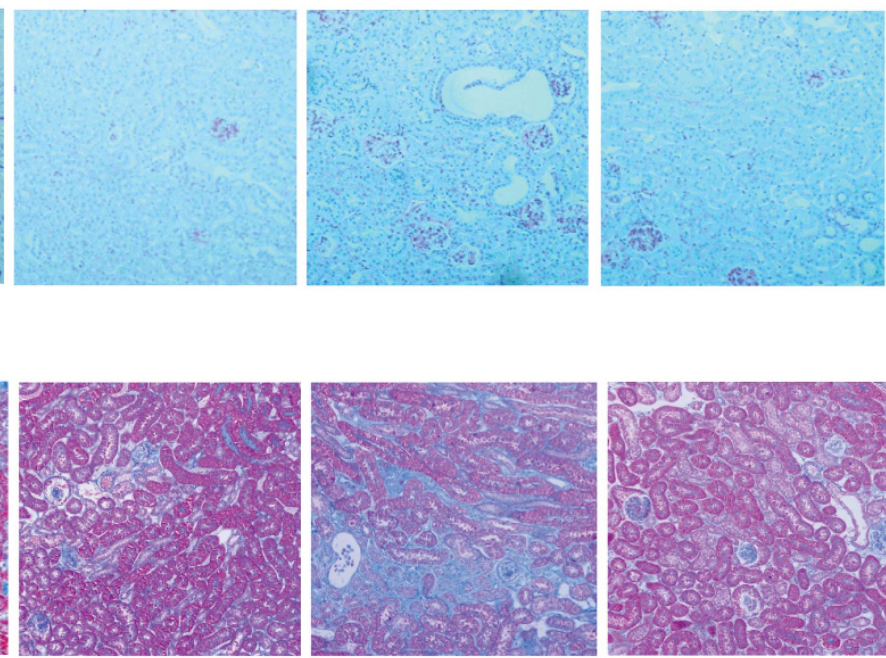

Ald-BM

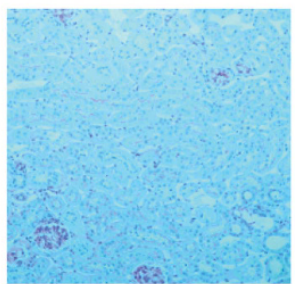

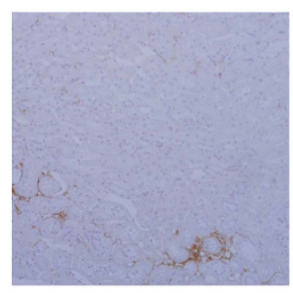

b
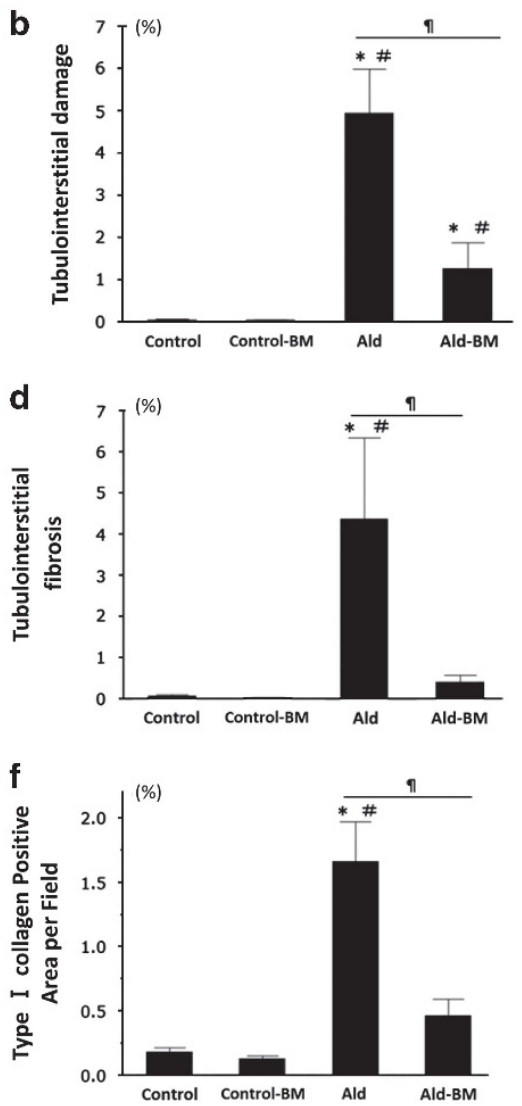

h

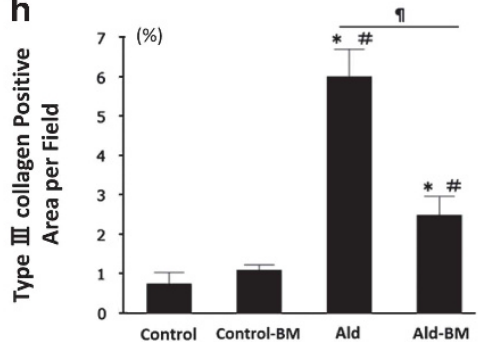

Figure 6 Histological analyses for tubular damage stained with periodic acid-Schiff (PAS) (a). Tubular damage was assessed quantitatively in PAS-stained sections (b). Tubulointerstitial fibrosis was stained with Masson Trichrome stain (c) and assessed quantitatively (d). Immunohistological staining for type $\alpha$ collagen (e) and type $\beta$ collagen (g); the positively stained areas were assessed quantitatively ( $\mathbf{f}$ and $\mathbf{h}$ ). Original magnification, $\times 100$. Values are mean \pm s.e. ${ }^{*} P<0.05$ vs the control group; ${ }^{\#} P<0.05$ vs the control-BM group; ${ }^{\top} P<0.05$ vs the Ald group. Abbreviations: Ald, aldosterone; BM, bardoxolone methyl.

Gene expressions of RANTES and type I and III collagens in the kidney

The mRNA level of RANTES was measured to evaluate the inflammatory response. The level was significantly higher in the Ald group than in the control and control-BM groups (Figure $5 \mathrm{a}$ ), and in the Ald-BM than in the control-BM group. The increase in RANTES level in the Ald group was significantly ameliorated in the Ald-BM group. The gene expression of RANTES was significantly lower in the control-BM group than in the control group.

The levels of $\alpha-1$ collagen type I and type III were measured to evaluate interstitium fibrosis. In the Ald group, the mRNA expressions of $\alpha-1$ collagen type I (Figure $5 b$ ) and type III (Figure $5 c$ ) were significantly higher than in the control and control-BM groups. Their expressions were significantly lower in the Ald-BM group than in the Ald group and the mRNA level of $\alpha-1$ collagen type III was significantly lower in the control-BM group than in the control group.

\section{Renal histological and morphometric analyses}

Although tubular damage as assessed by periodic acid-Schiff staining staining was significantly more severe in the Ald and Ald-BM groups than in the control and control-BM groups, it was significantly attenuated in the Ald-BM group compared with the Ald group $(P<0.05$, Figures $6 \mathrm{a}$ and $\mathrm{b})$. The tubulointerstitial fibrosis as assessed by Masson's trichrome staining was significantly more severe in the Ald group than in the control and control-BM groups, and was significantly attenuated in the Ald-BM group $(P<0.05$, Figures $6 c$ and d).

\section{Immunohistological analysis for type I and III collagens}

Areas of type I and III collagen deposition were observed in the interstitium (Figures $6 \mathrm{e}$ and $\mathrm{g}$ ). The areas positively stained for type I (Figure 6f) and type III (Figure 6h) collagens were significantly higher $(P<0.05)$ in the Ald group than in the control and control-BM 

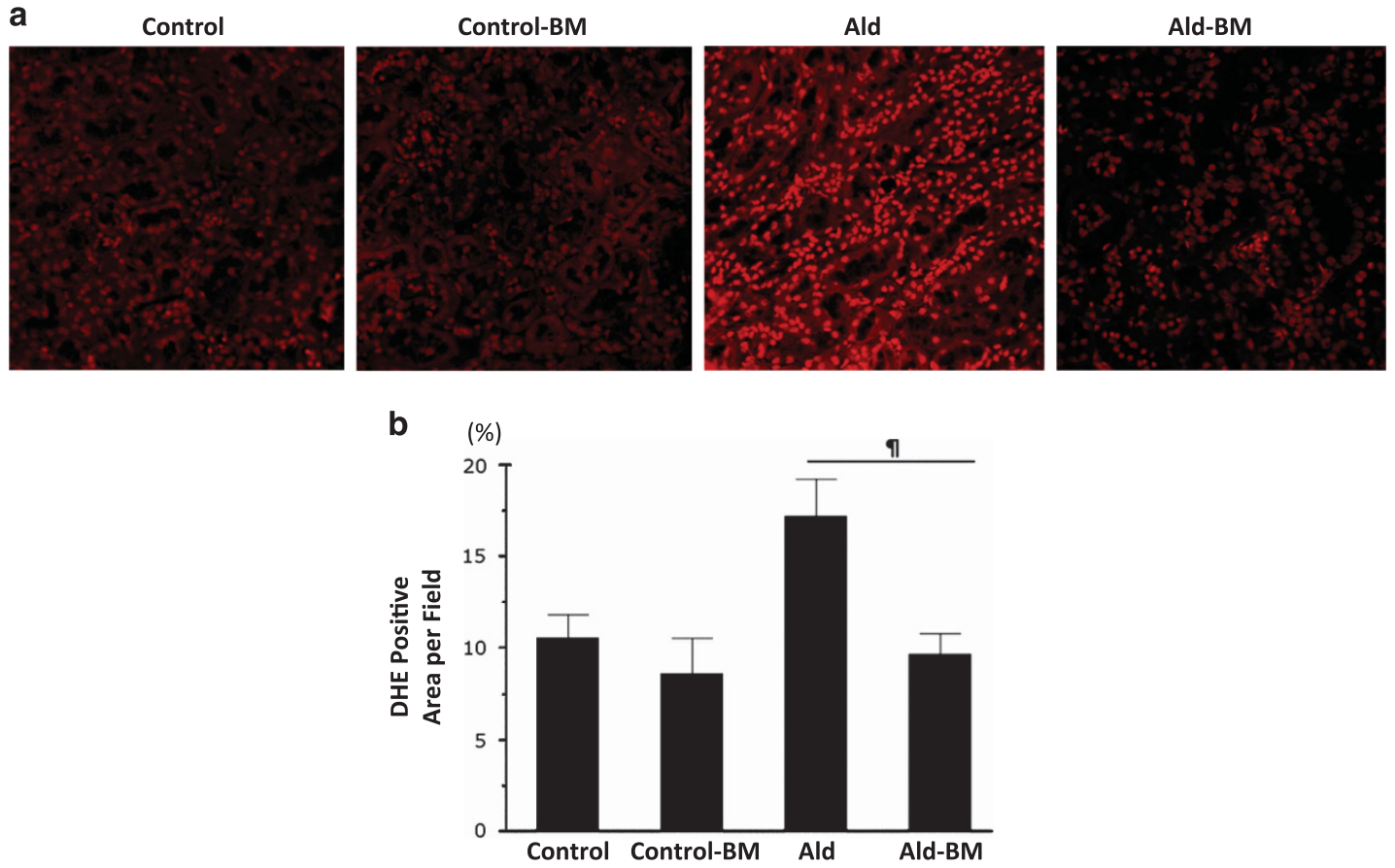

Figure 7 Immunofluorescent staining for $\mathrm{DHE}(\mathbf{a})$. The positively stained areas of $\mathrm{DHE}$ were assessed quantitatively (b). Values are mean $\pm \mathrm{S} . \mathrm{e}$. ${ }^{\star} P<0.05$ vs the control group; ${ }^{\#} P<0.05$ vs the control-BM group; ${ }^{\top} P<0.05$ vs the Ald group. Abbreviations: Ald, aldosterone; BM, bardoxolone methyl; DHE, dihydroethidium.

groups. Their deposition areas were significantly lower in the Ald-BM group compared with the Ald group, although the type III collagen deposition area was significantly higher in the Ald-BM group than in the control and control-BM groups.

\section{Evaluation of oxidative stress}

DHE staining was applied to the kidney sections to evaluate ROS production. DHE fluorescence tended to be greater in the Ald group than in the control group and was significantly lower in the Ald-BM group $(P<0.05$, Figures $7 \mathrm{a}$ and $\mathrm{b})$.

Gene and protein expressions in the kidneys and urinary excretion of human L-FABP

The mRNA levels of human L-FABP in the kidneys were significantly higher in the Ald group than in the control and control-BM groups and significantly lower in the Ald-BM group than in the Ald group. Furthermore, the levels in the Ald-BM group were significantly lower than in the control and control-BM groups (Figure 8a). The protein expressions of human L-FABP in the kidneys were significantly higher in the Ald group than in the control group, and significantly lower in the Ald-BM group (Figure 8b). Urinary human L-FABP levels on day 35 were significantly higher in the Ald group than in the control and control-BM groups and significantly higher in the Ald-BM group than in the control group (Figure $8 \mathrm{c}$ ). The levels were significantly lower in the Ald-BM group than in the Ald group $(P<0.05)$.

\section{Correlation between urinary L-FABP and the degree of} tubulointerstitial damage on day 35

Urinary L-FABP levels significantly correlated with the degree of tubulointerstitial damage as assessed by periodic acid-Schiff staining staining, infiltration of macrophage as evaluated using an antibody against $\mathrm{F} 4 / 80$ and tubulointerstitial fibrosis as indicated using antibodies against both type I and type III collagens (Figure 9).

\section{DISCUSSION}

This study revealed that administration of the Nrf2 activator BM attenuated the renal oxidative stress, excessive activation of the intrarenal RAS, inflammation and tubulointerstitial damage induced by both Ald and salt. Furthermore, the dynamics of human L-FABP changed with the administration of BM, with urinary human L-FABP levels reflecting the other changes caused by the BM.

Administering BM reduced the renal gene expression of Nrf2 in the present study. The BM bound to cysteine residues of Keap1 and leaded to conformational changes in Keap1 disrupting the interaction between Nrf2 and Keap1, resulting in inhibiting the degradation of the Nrf2 by polyubiquitination. ${ }^{31,32}$ We considered that because the renal Nrf2 protein expression was maintained at a higher level by BM, the mRNA expression of Nrf2 was suppressed. Therefore, the BM enhanced the renal expressions of the Nrf2 target antioxidant genes, which led to the suppression of ROS production as evaluated by DHE fluorescence and subsequently to significant attenuation of the tubulointerstitial damage induced by both Ald and salt (Figure 10).

We have previously reported a study of human L-FABP chromosomal transgenic mice in an Ald- and salt-induced hypertension model, which showed human L-FABP was upregulated in the proximal tubules and attenuated tubulointerstitial damage via antioxidative action, as compared to the wild-type mice without L-FABP expression in their proximal tubules. ${ }^{18}$ Although the duration of Ald-injection was longer in the present study (5 weeks) than in the previous study ( 4 weeks), ${ }^{18}$ the present study also demonstrated the upregulation of renal human L-FABP with Ald and salt, and that administration of $\mathrm{BM}$ suppressed this upregulation in the $\mathrm{FABP}^{+/-}$ mice. These results suggest that BM exhibited antioxidative action in human proximal tubules with L-FABP expression and may retard the progression of tubulointerstitial damage in clinical practice.

The present study demonstrated that systemic Nrf2 activation due to a chemical inducer of Nrf2 ameliorated tubulointerstitial damage in 
a

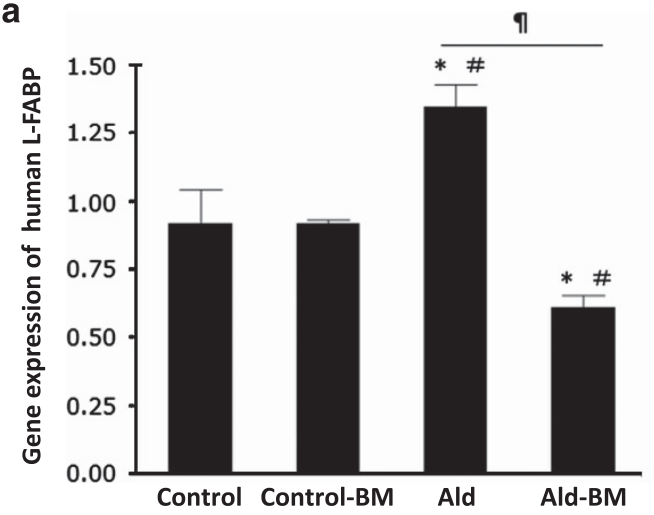

b

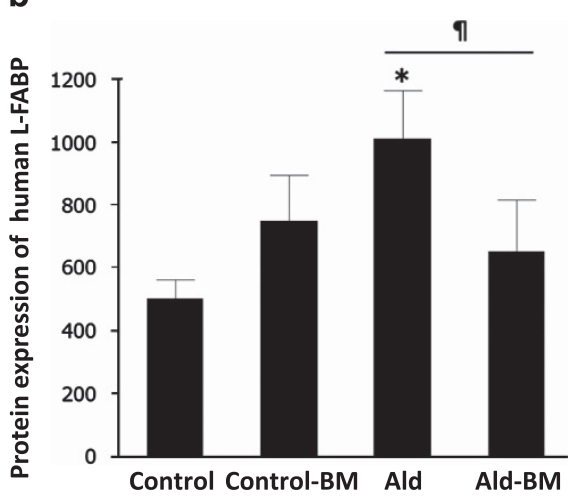

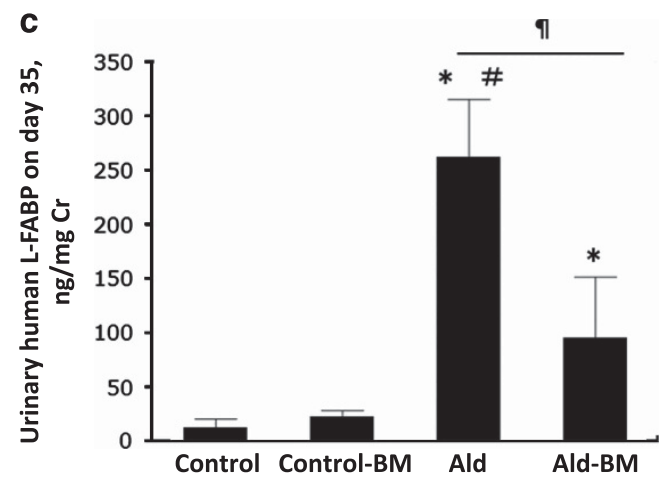

Figure 8 mRNA transcript expression (a) and protein expression (b) of human L-FABP. Urinary excretion of human L-FABP on day 35 (c). Values are mean \pm s.e. ${ }^{*} P<0.05$ vs the control group; $\# P<0.05$ vs the control-BM group; $\mathbb{\uparrow} P<0.05$ vs the Ald group. Abbreviations: Ald, aldosterone; BM, bardoxolone methyl; L-FABP, liver-type fatty acid-binding protein.

the Ald- and salt-induced hypertension model. The kidney is composed of various types of cells and pharmacological Nrf2 activation has been reported to work on glomerular mesangial cells $^{33}$ in addition to the tubules. Although significant glomerular damage was not induced in the Ald- and salt-induced hypertension mouse model ${ }^{18}$ and this study did not show an increase in the Nrf2 protein expression in the glomeruli (data not shown), it is possible that the increase in Nrf2 target antioxidant in these cells due to the $\mathrm{BM}$ influenced the improvement to the tubulointerstitial damage observed in the Ald-BM mice. To clarify this point requires a genetic approach with cell-specific Nrf2 deletion mice.

In the present study, SBP on day 35 tended to be lower in the Ald-BM mice than in the Ald mice, suggesting the possibility that BM influenced SBP. Some studies have reported that Nrf2 activators attenuate blood pressure elevation in hypertension models ${ }^{34,35}$ via regulation of myogenic tone due to increased expression of Nrf2-regulated antioxidant genes in vascular smooth muscle. ${ }^{24}$ As vascular Nrf2-antioxidant system may exert vasoprotective effects in hypertension-associated vascular changes induced by increased vascular oxidative stress, the effects of the BM may be related to the decrease in SBP by BM in the Ald- and salt-induced model. Although decrease in blood pressure is reported not to lead to attenuation of renal injury and reduction of oxidative stress in the Ald- and salt- induced renal injury model, ${ }^{18,36}$ such vasoprotective actions of BM may be useful for the improvement of vascular function in hypertension.

In contrast to the renoprotective effect of $\mathrm{BM}$, a pathogenic role of Nrf2 in inflammasome activation has been reported. ${ }^{37,38}$ Freigang et al. $^{37}$ reported that an $\mathrm{Nrf2}$ activator was a positive regulator of the inflammasome and enhanced interleukin-1-mediated vascular inflammation. Zhao et al. ${ }^{38}$ also reported that Nrf2 mediated inflammasome activation, leading to an increase in inflammatory response. Administration of an initial daily dose of BM of $10 \mathrm{mg} \mathrm{kg}^{-1}$ induced high mouse mortality in our preliminary study. A clinical study of treatment with BM for stage 4 CKD patients with diabetes mellitus showed that it significantly increased the risk of heart failure and of a composite cardiovascular outcome (nonfatal myocardial infarction, nonfatal stroke, hospitalization for heart failure or death from cardiovascular causes). ${ }^{39}$ Zoja et al. ${ }^{40}$ demonstrated the divergent result of Nrf2 activator RTA405, a synthetic triterpenoid analog of BM, against renoprotective effect using Zucker diabetic fatty rats. ${ }^{40}$ Furthermore, they also showed acute liver toxicity of RTA405 in the diabetic rats. As the roles of Nrf2 and Nrf2 activators differ between various pathophysiological conditions, further studies are needed to examine the utility of BM for each disease and severity of disease to establish when it can be applied in clinical practice.

Urinary human L-FABP is a tubular marker that accurately reflects the degree of tubulointerstitial damage and has the potential to predict prognosis in CKD. ${ }^{17,41}$ In a clinical study of BM, urinary albumin did not change in line with the treatment effect of $\mathrm{BM} ;{ }^{23}$ it was speculated that $\mathrm{BM}$ reduced the expression of megalin in the proximal tubules, and that this was related to reabsorbing the albumin filtered through glomeruli. ${ }^{42}$ In the present study, urinary L-FABP levels significantly correlated with the degree of tubulointerstitial damage. Although urinary albumin levels decreased in response to the administration of $\mathrm{BM}$ and this study did not demonstrate the superiority of urinary human L-FABP over urinary albumin for evaluating the renoprotective effect of BM, urinary L-FABP may be useful for determining the effect of BM in clinical practice. 

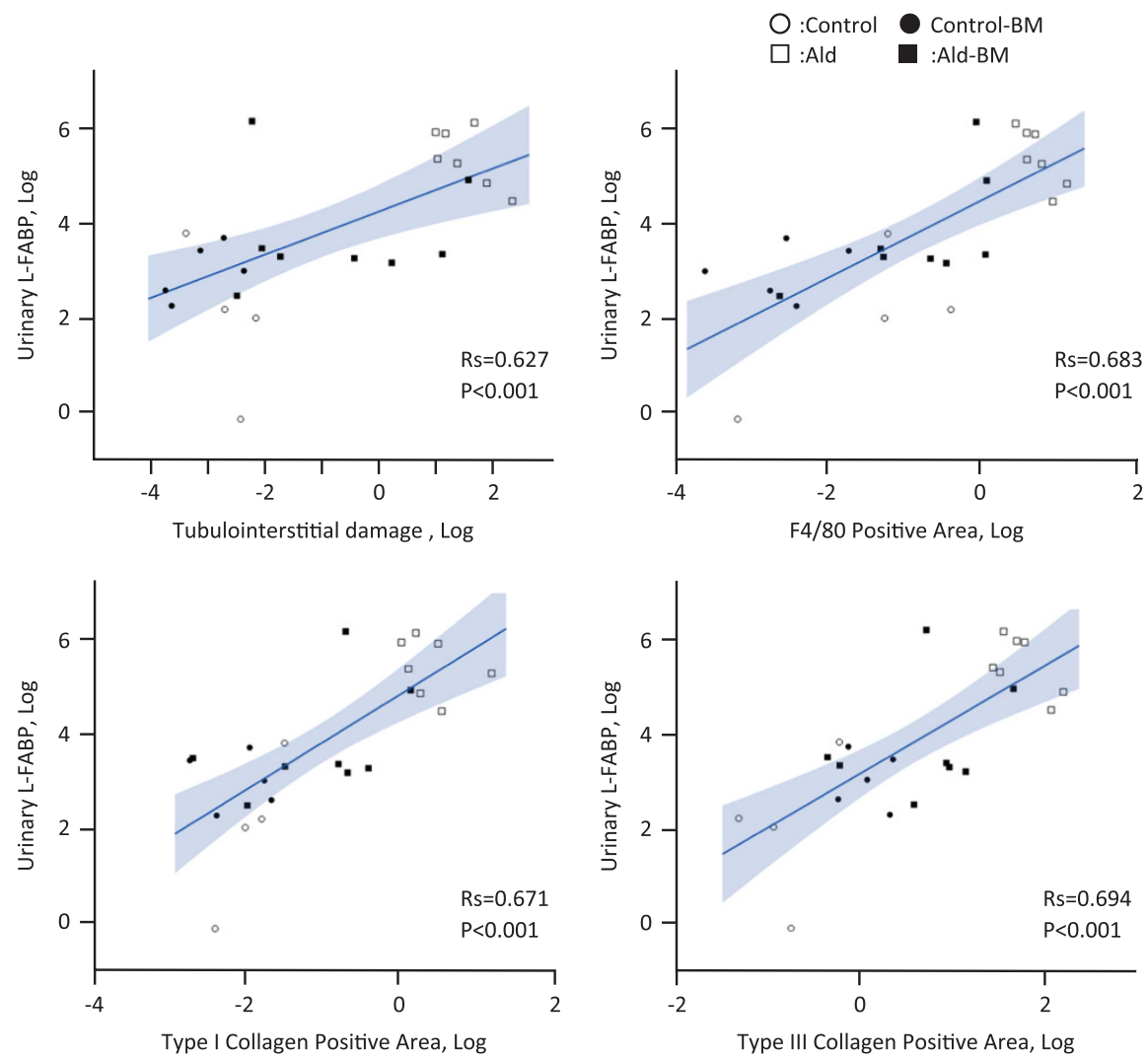

Figure 9 Correlations between urinary L-FABP and each histological marker on day 35. White circle ( 0 ) shows control group, black circle ( $\bullet$ ) shows controlBM group, white square $(\square)$ shows Ald group and black square ( $\square$ ) shows Ald-BM group. Abbreviations: Ald, aldosterone; BM, bardoxolone methyl; L-FABP, liver-type fatty acid-binding protein.

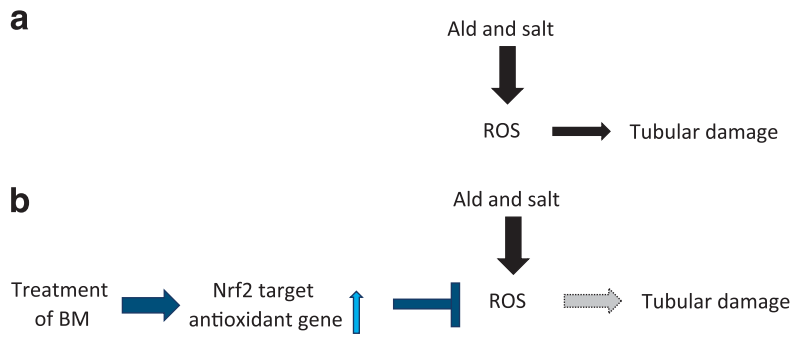

Figure 10 Mechanism for renal tubular damage in the Ald- and salt-induced hypertension model. Reactive oxygen species (ROS) produced by the Ald and salt in the proximal tubules leads to the progression of tubular damage (a). Treatment with BM upregulates the renal expression of the Nrf2 target antioxidant genes and suppresses ROS production, resulting in the attenuation of the tubular damage induced by the Ald and salt (b). Abbreviations: Ald, aldosterone; BM, bardoxolone methyl; Nrf2, nuclear factor erythroid 2-related factor 2 .

There is a critical limitation in this study. As urinary sodium levels were significantly lower in the Ald-BM group than in the Ald group, there is a possibility that $1 \% \mathrm{NaCl}$ water intake in the Ald-BM group significantly decreased compared with that in the Ald group, which may be related to renoprotection in the Ald-BM group. Zoja et al. ${ }^{40}$ also reported that BM analog RTA405 caused decreases in food intake and diuresis in rats with diabetic nephropathy. However, because severe tubulointerstitial damage causes a decrease in the reabsorption of urinary sodium, suppression of tubulointerstitial damage in the Ald-BM group may lead to the decrease in urinary excretion of sodium. In fact, serum sodium concentration in the Ald-BM group was higher than that in the Ald group. Further studies are needed to clarify this point.

In conclusion, this study showed that treatment with BM suppressed the progression of tubulointerstitial damage in Ald- and salt-induced hypertension under the expression of human L-FABP in the proximal tubules. Recent clinical studies in hypertensive CKD have reported RAS inhibitors to have only a limited effect on the suppression of renal dysfunction ${ }^{43}$ and $\mathrm{BM}$ may be expected to be part of a new strategy for preventing tubulointerstitial damage due to salt-sensitive hypertension. Furthermore, urinary L-FABP is a useful tubular marker that reflects the renoprotective effect of BM.

\section{CONFLICT OF INTEREST}

TS is the director and senior scientist of CMIC HOLDINGS Co., Ltd., the company that produced the kits for L-FABP analysis. All other authors declare no conflicts of interest.

\section{ACKNOWLEDGEMENTS}

We thank Ms. Kimie Katayama, Ms. Jyunko Asano and Mr. Kazuho Inoue for technical assistance. This research did not receive any specific grant from funding agencies in the public, commercial or not-for-profit sectors.

1 Fujita T. Mineralocorticoid receptors, salt-sensitive hypertension, and metabolic syndrome. Hypertension 2010; 55: 813-818.

2 Blasi ER, Rocha R, Rudolph AE, Blomme EA, Polly ML, McMahon EG. Aldosterone/salt induces renal inflammation and fibrosis in hypertensive rats. Kidney Int 2003; 63: 1791-1800. 
3 Kawarazaki W, Nagase M, Yoshida S, Takeuchi M, Ishizawa K, Ayuzawa N, Ueda K, Fujita T. Angiotensin II- and salt-induced kidney injury through Rac1-mediated mineralocorticoid receptor activation. J Am Soc Nephrol 2012; 23: 997-1007.

4 Nagase M, Shibata S, Yoshida S, Nagase T, Gotoda T, Fujita T. Podocyte injury underlies the glomerulopathy of Dahl salt-hypertensive rats and is reversed by aldosterone blocker. Hypertension 2006; 47: 1084-1093.

5 Ando K, Ohtsu H, Uchida S, Kaname S, Arakawa Y, Fujita T. Group ES: Anti-albuminuric effect of the aldosterone blocker eplerenone in non-diabetic hypertensive patients with albuminuria: a double-blind, randomised, placebo-controlled trial. Lancet Diabetes Endocrinol 2014; 2: 944-953.

6 Navaneethan SD, Nigwekar SU, Sehgal AR, Strippoli GF. Aldosterone antagonists for preventing the progression of chronic kidney disease: a systematic review and metaanalysis. Clin J Am Soc Nephrol 2009; 4: 542-551.

7 Irita J, Okura T, Jotoku M, Nagao T, Enomoto D, Kurata M, Desilva VR, Miyoshi K, Matsui Y, Uede T, Denhardt DT, Rittiling SR, Higaki J. Osteopontin deficiency protects against aldosterone-induced inflammation, oxidative stress, and interstitial fibrosis in the kidney. Am J Physiol Renal Physiol 2011; 301: F833-F844.

8 Nagase M, Fujita T. Role of Rac1-mineralocorticoid-receptor signalling in renal and cardiac disease. Nat Rev Nephrol 2013; 9: 86-98.

9 Kiyomoto H, Rafiq K, Mostofa M, Nishiyama A. Possible underlying mechanisms responsible for aldosterone and mineralocorticoid receptor-dependent renal injury. J Pharm Sci 2008; 108: 399-405.

10 Nishiyama A, Yao L, Nagai Y, Miyata K, Yoshizumi M, Kagami S, Kondo S, Kiyomoto H, Shokoji T, Kimura S, Kohno M, Abe Y. Possible contributions of reactive oxygen species and mitogen-activated protein kinase to renal injury in aldosterone/salt-induced hypertensive rats. Hypertension 2004; 43: 841-848.

11 Shibata S, Nagase M, Yoshida S, Kawachi H, Fujita T. Podocyte as the target for aldosterone: roles of oxidative stress and Sgk1. Hypertension 2007; 49: 355-364.

12 Sun GP, Kohno M, Guo P, Nagai Y, Miyata K, Fan YY, Kimura S, Kiyomoto H, Ohmori K, Li DT, Abe Y, Nishiyama A. Involvements of Rho-kinase and TGF-beta pathways in aldosterone-induced renal injury. J Am Soc Nephrol 2006; 17: 2193-2201.

13 Prunotto M, Budd DC, Gabbiani G, Meier M, Formentini I, Hartmann G, Pomposiello S, Moll S. Epithelial-mesenchymal crosstalk alteration in kidney fibrosis. J Pathol 2012 228: 131-147.

14 Remuzzi G, Bertani T. Pathophysiology of progressive nephropathies. N Engl J Med 1998; 339: 1448-1456.

15 Yan J, Gong Y, She YM, Wang G, Roberts MS, Burczynski FJ. Molecular mechanism of recombinant liver fatty acid binding protein's antioxidant activity. J Lipid Res 2009; 50: 2445-2454.

16 Simon TC, Roth KA, Gordon JI. Use of transgenic mice to map cis-acting elements in the liver fatty acid-binding protein gene (Fabpl) that regulate its cell lineage-specific, differentiation-dependent, and spatial patterns of expression in the gut epithelium and in the liver acinus. J Biol Chem 1993; 268: 18345-18358.

17 Kamijo A, Sugaya T, Hikawa A, Okada M, Okumura F, Yamanouchi M, Honda A, Okabe M, Fujino T, Hirata Y, Omata M, Kaneko R, Fujii H, Fukamizu A, Kimura K. Urinary excretion of fatty acid-binding protein reflects stress overload on the proximal tubules. Am J Pathol 2004; 165: 1243-1255.

18 Ichikawa D, Kamijo-Ikemori A, Sugaya T, Shibagaki Y, Yasuda T, Hoshino S, Katayama K, Igarashi-Migitaka J, Hirata K, Kimura K. Human liver-type fatty acidbinding protein protects against tubulointerstitial injury in aldosterone-induced renal injury. Am J Physiol Renal Physiol 2015; 308: F114-F121.

19 Lee JM, Li J, Johnson DA, Stein TD, Kraft AD, Calkins MJ, Jakel RJ, Johnson JA. Nrf2, a multi-organ protector? FASEB J 2005; 19: 1061-1066.

20 Yoh K, Hirayama A, Ishizaki K, Yamada A, Takeuchi M, Yamagishi S, Morito N Nakano T, Ojima M, Shimohata H, Itoh K, Takahashi S, Yamamoto M. Hyperglycemia induces oxidative and nitrosative stress and increases renal functional impairment in Nrf2-deficient mice. Genes Cells 2008; 13: 1159-1170.

21 Yoh K, Itoh K, Enomoto A, Hirayama A, Yamaguchi N, Kobayashi M, Morito N, Koyama A, Yamamoto M, Takahashi S. Nrf2-deficient female mice develop lupus-like autoimmune nephritis. Kidney Int 2001; 60: 1343-1353.

22 Nezu M, Souma T, Yu L, Suzuki T, Saigusa D, Ito S, Suzuki N, Yamamoto M. Transcription factor Nrf2 hyperactivation in early-phase renal ischemia-reperfusion injury prevents tubular damage progression. Kidney Int 2017; 91: 387-401.

23 Pergola PE, Raskin P, Toto RD, Meyer CJ, Huff JW, Grossman EB, Krauth M, Ruiz S, Audhya P, Christ-Schmidt H, Wittes J, Warnock DGInvestigators BS. Bardoxolone methyl and kidney function in CKD with type 2 diabetes. N Engl J Med 2011; 365: 327-336.

24 Lopes RA, Neves KB, Tostes RC, Montezano AC, Touyz RM. Downregulation of nuclear factor erythroid 2-related factor and associated antioxidant genes contributes to redox-sensitive vascular dysfunction in hypertension. Hypertension 2015; 66: 1240-1250

25 Ichikawa D, Kamijo-Ikemori A, Sugaya T, Yasuda T, Hoshino S, Igarashi-Migitaka J, Hirata K, Kimura K. Renal liver-type fatty acid binding protein attenuates angiotensin II-induced renal injury. Hypertension 2012; 60: 973-980.

26 Matsui K, Kamijo-Ikemori A, Sugaya T, Yasuda T, Kimura K. Renal liver-type fatty acid binding protein (L-FABP) attenuates acute kidney injury in aristolochic acid nephrotoxicity. Am J Pathol 2011; 178: 1021-1032.

27 Yokoyama T, Kamijo-Ikemori A, Sugaya T, Hoshino S, Yasuda T, Kimura K. Urinary excretion of liver type fatty acid binding protein accurately reflects the degree of tubulointerstitial damage. Am J Pathol 2009; 174: 2096-2106.
28 Inoue D, Suzuki T, Mitsuishi Y, Miki Y, Suzuki S, Sugawara S, Watanabe M, Sakurada A, Endo C, Uruno A, Sasano H, Nakagawa T, Satoh K, Tanaka N, Kubo H, Motohashi H, Yamamoto M. Accumulation of p62/SQSTM1 is associated with poor prognosis in patients with lung adenocarcinoma. Cancer Sci 2012; 103: 760-766.

29 Ichikawa D, Kamijo-Ikemori A, Sugaya T, Shibagaki Y, Yasuda T, Katayama K, Hoshino S, Igarashi-Migitaka J, Hirata K, Kimura K. Renoprotective effect of renal liver-type fatty acid binding protein and angiotensin II type la receptor loss in renal injury caused by RAS activation. Am J Physiol Renal Physiol 2014; 306: F655-F663.

30 Lopez-Parra V, Mallavia B, Lopez-Franco O, Ortiz-Munoz G, Oguiza A, Recio C, Blanco J, Nimmerjahn F, Egido J, Gomez-Guerrero C. Fcgamma receptor deficiency attenuates diabetic nephropathy. J Am Soc Nephrol 2012; 23: 1518-1527.

31 Sporn MB, Liby KT, Yore MM, Fu L, Lopchuk JM, Gribble GW. New synthetic triterpenoids: potent agents for prevention and treatment of tissue injury caused by inflammatory and oxidative stress. J Nat Prod 2011; 74: 537-545.

32 Yates MS, Tauchi M, Katsuoka F, Flanders KC, Liby KT, Honda T, Gribble GW, Johnson DA, Johnson JA, Burton NC, Guilarte TR, Yamamoto M, Sporn MB, Kensler TW. Pharmacodynamic characterization of chemopreventive triterpenoids as exceptionally potent inducers of Nrf2-regulated genes. Mol Cancer Ther 2007; 6: 154-162.

33 Ding Y, Stidham RD, Bumeister R, Trevino I, Winters A, Sprouse M, Ding M, Ferguson DA, Meyer CJ, Wigley WC, Ma R. The synthetic triterpenoid, RTA 405, increases the glomerular filtration rate and reduces angiotensin II-induced contraction of glomerular mesangial cells. Kidney Int 2013; 83: 845-854.

34 Senanayake GV, Banigesh A, Wu L, Lee P, Juurlink BH. The dietary phase 2 protein inducer sulforaphane can normalize the kidney epigenome and improve blood pressure in hypertensive rats. Amournal J Hypertens 2012; 25: 229-235.

35 Wu L, Noyan Ashraf MH, Facci M, Wang R, Paterson PG, Ferrie A, Juurlink BH. Dietary approach to attenuate oxidative stress, hypertension, and inflammation in the cardiovascular system. Proc Natl Acad Sci USA 2004; 101: 7094-7099.

36 Sawada H, Naito Y, Oboshi M, Iwasaku T, Okuhara Y, Morisawa D, Eguchi A, Hirotani S, Masuyama T. Iron restriction inhibits renal injury in aldosterone/salt-induced hypertensive mice. Hypertens Res 2015; 38: 317-322.

37 Freigang S, Ampenberger F, Spohn G, Heer S, Shamshiev AT, Kisielow J, Hersberger M, Yamamoto M, Bachmann MF, Kopf M. Nrf2 is essential for cholesterol crystal-induced inflammasome activation and exacerbation of atherosclerosis. Eur J Immunol 2011; 41. 2040-2051.

38 Zhao C, Gillette DD, Li X, Zhang Z, Wen H. Nuclear factor E2-related factor-2 (Nrf2) is required for NLRP3 and AIM2 inflammasome activation. J Biol Chem 2014; 289: $17020-17029$.

39 deZeeuw D, Akizawa T, Audhya P, Bakris GL, Chin M, Christ-Schmidt H, Goldsberry A, Houser M, Krauth M, Lambers Heerspink HJ, McMurray JJ, Meyer CJ, Parving HH, Remuzzi G, Toto RD, Vaziri ND, Wanner C, Wittes J, Wrolstad D, Chertow GM, Investigators BT. Bardoxolone methyl in type 2 diabetes and stage 4 chronic kidney disease. N Engl J Med 2013; 369: 2492-2503.

40 Zoja C, Corna D, Nava V, Locatelli M, Abbate M, Gaspari F, Carrara F, Sangalli F, Remuzzi G, Benigni A. Analogs of bardoxolone methyl worsen diabetic nephropathy in rats with additional adverse effects. Am J Physiol Renal Physiol 2013; 304: F808-F819.

41 Kamijo A, Kimura K, Sugaya T, Yamanouchi M, Hikawa A, Hirano N, Hirata Y, Goto A Omata $\mathrm{M}$. Urinary fatty acid-binding protein as a new clinical marker of the progression of chronic renal disease. J Lab Clin Med 2004; 143: 23-30.

42 Reisman SA, Chertow GM, Hebbar S, Vaziri ND, Ward KW, Meyer CJ. Bardoxolone methyl decreases megalin and activates nrf2 in the kidney. J Am Soc Nephrol 2012; 23: 1663-1673.

43 Appel LJ, Wright JT Jr., Greene T, Kusek JW, Lewis JB, Wang X, Lipkowitz MS, Norris KC, Bakris GL, Rahman M, Contreras G, Rostand SG, Kopple JD, Gabbai FB, Schulman GI, Gassman JJ, Charleston J, Agodoa LY, African American Study of Kidney D, Hypertension Collaborative Research G. Long-term effects of reninangiotensin system-blocking therapy and a low blood pressure goal on progression of hypertensive chronic kidney disease in African Americans. Arch Intern Med 2008; 168: 832-839.

(i) () $\odot$ This work is licensed under a Creative Commons Attribution-NonCommercial-NoDerivs 4.0 International License. The images or other third party material in this article are included in the article's Creative Commons license, unless indicated otherwise in the credit line; if the material is not included under the Creative Commons license, users will need to obtain permission from the license holder to reproduce the material. To view a copy of this license, visit http://creativecommons.org/licenses/bync-nd/4.0/

(C) The Author(s) 2018 